\title{
The Best Approximation Theorems and Fixed Point Theorems for Discontinuous Increasing Mappings in Banach Spaces
}

\author{
Dezhou Kong, ${ }^{1,2}$ Lishan Liu, ${ }^{1,3}$ and Yonghong $\mathrm{Wu}^{3}$ \\ ${ }^{1}$ School of Mathematical Sciences, Qufu Normal University, Qufu, Shandong 273165, China \\ ${ }^{2}$ College of Information Sciences and Engineering, Shandong Agricultural University, Taian, Shandong 271018, China \\ ${ }^{3}$ Department of Mathematics and Statistics, Curtin University of Technology, Perth, WA 6845, Australia
}

Correspondence should be addressed to Lishan Liu; mathlls@163.com

Received 20 June 2014; Revised 30 September 2014; Accepted 30 September 2014

Academic Editor: Poom Kumam

Copyright (C) 2015 Dezhou Kong et al. This is an open access article distributed under the Creative Commons Attribution License, which permits unrestricted use, distribution, and reproduction in any medium, provided the original work is properly cited.

\begin{abstract}
We prove that Fan's theorem is true for discontinuous increasing mappings $f$ in a real partially ordered reflexive, strictly convex, and smooth Banach space $X$. The main tools of analysis are the variational characterizations of the generalized projection operator and order-theoretic fixed point theory. Moreover, we get some properties of the generalized projection operator in Banach spaces. As applications of our best approximation theorems, the fixed point theorems for non-self-maps are established and proved under some conditions. Our results are generalizations and improvements of the recent results obtained by many authors.
\end{abstract}

\section{Introduction}

Let $X$ be a real Banach space with the dual space $X^{*}$ and $C \subset$ $X$ a nonempty subset of $X$. The set-valued mapping $P_{C}: X \rightarrow$ C,

$$
P_{C}(x)=\left\{z \in C:\|x-z\|=\inf _{y \in C}\|x-y\|\right\},
$$

is called the metric projection operator from $X$ onto $C$. It is well known that the metric projection operator $P_{C}$ plays an important role in nonlinear functional analysis, optimization theory, fixed point theory, nonlinear programming, game theory, variational inequality, complementarity problems, and so forth.

In 1994, Alber [1] introduced the generalized projections $\pi_{C}: X^{*} \rightarrow C$ and $\Pi_{C}: X \rightarrow C$ from Hilbert spaces to uniformly convex and uniformly smooth Banach spaces and studied their properties in detail. In [2], Li extended the generalized projection operator $\pi_{C}$ from uniformly convex and uniformly smooth Banach spaces to reflexive Banach spaces and studied some properties of the generalized projection operator with applications to solving the variational inequality in Banach spaces. Recently, Isac [3] and Nishimura and Ok [4] studied the order-theoretic approach towards establishing the solvability of variational inequality on a Hilbert lattice $X$ which is based on the fact that the metric projection operator $P_{C}$ is order-preserving if only if $C$ is a sublattice of $X$. Very recently, Li and Ok [5] obtained the generalized projection operator $\pi_{C}$ is order-preserving in partially ordered Banach spaces.

Motivated and inspired by the above mentioned work, in this paper, we get the continuous property of generalized projection operator $\Pi_{C}$ and increasing characterizations of $\Pi_{C}$ in a partially ordered reflexive, strict convex, and smooth Banach space. Further, we consider the following Fan's approximation theorem (Theorem 2 in [6]) through the variational characterization of $\Pi_{C}$. The normed space version of the theorem is as follows.

Theorem 1. Let $C$ be a nonempty compact convex set in a normed linear space $X$. If $f$ is a continuous map from $C$ into $X$, then there exists a point $u$ in $C$ such that

$$
\|u-f(u)\|=d(f(u), C) .
$$

The point $u$ is called a best approximation point of $f$ in $C$.

Fan's theorem has been of great importance in nonlinear analysis, approximation theory, game theory, and minimax 
theorems. Various aspects of this theorem have been studied by many authors under different assumptions. For some related works, refer to [7-21] and the references therein.

In this paper, we obtain the existence of minimum best approximation point and maximum best approximation point in order interval. As an applications of our best approximation theorems, the fixed point theorems for nonself-maps are established under some conditions which do not need to require any continuous and compact conditions on $f$.

The content of the present work can be summarized as follows. In Section 2, we review the definition of the generalized projection operator in Banach spaces and its basic properties. We also show some definitions in the partially ordered Banach space and some fundamental results for our theorems. In Section 3, we obtain the properties of the generalized projection operator in the partially ordered Banach space under some assumption. And we combine these results with an order-theoretic fixed point theorem to provide some of the best approximation theorems. Section 4 provides an application of these best approximation theorems to fixed point theory.

\section{Preliminaries}

2.1. The Partial Order. Suppose that $X$ is a real Banach space and $P$ is a nonempty closed convex cone of $X$. By $\theta$ we denote the zero element of $X$. We define a partial order $\preceq$ with respect to $P$ by $x \preceq y$ if and only if $y-x \in P$. We will write $x \prec y$ if $x \preceq y$ and $x \neq y$.

The cone $P$ is called normal if there is a number $K>0$, such that for all $x, y \in X, 0 \preceq x \preceq y$ implies $\|x\| \leq K\|y\|$. The cone $P$ is called regular if every increasing sequence which is bounded from above is convergent. That is, if $\left\{x_{n}\right\}$ is a sequence such that $x_{1} \preceq x_{2} \preceq \cdots \preceq y$ for some $y \in X$, then there is $x \in X$ such that $\lim _{n \rightarrow \infty}\left\|x_{n}-x\right\|=0$. Equivalently, the cone $P$ is regular if and only if every decreasing sequence which is bounded from below is convergent. It has been proved in Theorem 1.2.1 in [22] that every regular cone is normal.

A cone $P$ is called minihedral, if each two-element set $\{x, y\}$ has a least upper bound $\sup \{x, y\}$. Equivalently, the cone $P$ is minihedral if and only if each two-element set $\{x, y\}$ has a greatest lower bound $\inf \{x, y\}$. As is convenient, we denote $\sup \{x, y\}$ as $x \vee y$ and $\inf \{x, y\}$ as $x \wedge y$. And if $\sup M$ exists for every nonempty and bounded from above $M \subset X$, we say the cone $P$ is a strongly minihedral cone. If $M$ is a nonempty subset of $X$ which contains $x \vee y$ and $x \wedge y$ for every $x, y \in M$, then $M$ is said to be subminihedral.

Let $(X, \preceq)$ be a real partially ordered Banach space. Given $u_{0}, v_{0} \in X$ such that $u_{0} \prec v_{0}$, the set $\left[u_{0}, v_{0}\right]=\left\{z \in X: u_{0} \preceq\right.$ $\left.z \preceq v_{0}\right\}$ is called ordered interval. If the cone $P$ is minihedral, it is easy to see that $\left[u_{0}, v_{0}\right]$ is a subminihedral set of $X$.

Definition 2 (see [5]). For any partially ordered spaces $\left(X, \unlhd_{X}\right)$ and $\left(Y, \preceq_{Y}\right)$, we say that a map $F: X \rightarrow Y$ is orderpreserving if

$$
x \preceq_{X} y \text { implies } F(x) \preceq_{Y} F(y) .
$$

Definition 3 (see [23]). Let $(X, \preceq)$ be a partially ordered space and $D \subset X$ is convex; we say that a map $F: D \rightarrow X$ is convex if

$$
\begin{array}{r}
F(t x+(1-t) y) \preceq t F(x)+(1-t) F(y), \\
\forall x, y \in D, \quad x \preceq y, \quad 0 \leq t \leq 1 .
\end{array}
$$

2.2. Order-Dual. Let $(X, \preceq)$ be a real partially ordered Banach space whose (topological) dual we denote by $X^{*}$ and $P$ a cone in $X$. Recall that $P^{*}=\left\{\phi \in X^{*}: \phi(x) \geq 0, \forall x \in P\right\}$ is called the dual cone of $P$. The dual of $\preceq$ is the partial order ${ }^{*}$ on $X^{*}$ defined as follows:

$$
\phi \preceq^{*} \varphi \quad \text { iff } \varphi-\phi \in P^{*} .
$$

If $P$ is a minihedral cone, it is well known that $P^{*}$ is a minihedral cone in $X^{*}$. We now show that $x \in P$ if and only if $\langle\varphi, x\rangle \geq 0$ for every $\varphi \in P^{*}$ (see [24, Proposition 1.4.2]).

We denote by $\left(H,\|\cdot\|_{1}\right)$ a Hilbert space $H$ whose norm $\|\cdot\|_{1}$ satisfies

$$
|x| \preceq|y| \text { implies }\|x\|_{1} \leq\|y\|_{1}, \quad \forall x, y \in H,
$$

where $|x|$ is defined by $|x|=x \vee(-x)$ for each $x \in H$.

2.3. The Generalized Projection Operator. Let $X$ be a real Banach space with the dual $X^{*}$. We denote by $J$ the normalized duality mapping from $X$ to $2^{X^{*}}$ defined by

$$
J x=\left\{x^{*} \in X^{*}:\left\langle x^{*}, x\right\rangle=\left\|x^{*}\right\|\|x\|,\|x\|=\left\|x^{*}\right\|\right\},
$$

for all $x \in X$, where $\langle\cdot, \cdot\rangle$ denotes the generalized duality pairing between $X^{*}$ and $X$. See [1] for basic characterizations of the normalized duality mapping.

Recall that a Banach space $X$ has the Kadec-Klee property, if for any sequence $\left\{x_{n}\right\} \subset X$ and $x \in X$ with $x_{n} \rightarrow x$ (weak convergence) and $\left\|x_{n}\right\| \rightarrow\|x\|$, then $\left\|x_{n}-x\right\| \rightarrow 0$, as $n \rightarrow$ $\infty$. It is well known that if $X$ is a uniformly convex Banach space, then $X$ has the Kadec-Klee property.

Let $X$ be a reflexive, strictly convex, and smooth Banach space and $C$ a nonempty closed convex subset of $X$. Consider the Lyapunov functional defined by

$$
W(x, y)=\|x\|^{2}-2\langle J x, y\rangle+\|y\|^{2}, \quad \forall x, y \in X .
$$

Following Alber [1], the generalized projection $\Pi_{C}: X \rightarrow C$ is a map that assigns to an arbitrary point $x \in X$ the minimum point of the functional $W(x, y)$; that is, $\Pi_{C}(x)=\widehat{x}$, where $\widehat{x} \in C$ is the solution to the minimization problem:

$$
W(x, \widehat{x})=\inf _{y \in C} W(x, y) ;
$$

existence and uniqueness of the operator $\Pi_{C}$ follow from the properties of the functional $W(x, y)$ and strict monotonicity of the mapping $J$. It is obvious from the definition of functional $W$ that

$$
\begin{array}{r}
(\|x\|-\|y\|)^{2} \leq W(x, y) \leq(\|x\|+\|y\|)^{2}, \\
\forall x, y \in X .
\end{array}
$$

If $X$ is a Hilbert space, then $W(x, y)=(\|x-y\|)^{2}$ and $\Pi_{C}=P_{C}$. 
If $X$ is a reflexive, strictly convex, and smooth Banach space, then for $x, y \in X, W(x, y)=0$ if and only if $x=y$. It is sufficient to show that if $W(x, y)=0$ then $x=y$. From (10), we have $\|x\|=\|y\|$. This implies that $\langle J x, y\rangle=\|y\|^{2}=\|J x\|^{2}$. From the definition of $J$, one has $J x=J y$. Therefore, we have $x=y$. See $[25,26]$ for more details.

In [1], the generalized projection operators on arbitrary convex closed sets $C$ satisfy the following property.

The point $\Pi_{C}(x)=\widehat{x}$ is a generalized projection of $x$ on $C \subset X$ if and only if the following inequality is satisfied:

$$
\langle J x-J \hat{x}, \widehat{x}-y\rangle \geq 0, \quad \forall y \in C .
$$

We denote $d_{W}(x, C)=\inf \{W(x, y): y \in C\}$, where $x \in$ $X$ and $W$ is Lyapunov functional in $X$.

\section{Best Approximation Theorems}

First we give the following properties of the generalized projection operators.

Lemma 4 (see [27]). Let $(X, \preceq)$ be a real partially ordered reflexive, strictly convex, and smooth Banach space with respect to a minihedral cone P. Suppose $P^{*}$ is the dual cone of $P$. The following statements are equivalent:

$\left(H_{1}\right)$ the normalized duality mapping $J$ is order-preserving;

$\left(H_{2}\right)\|J x \wedge J y\|^{2}+\|J x \vee J y\|^{2} \leq\|x\|^{2}+\|y\|^{2}, \forall x, y \in X, x \preceq$ $y$.

Lemma 5 (see [27]). Let $(X, \preceq)$ be a real partially ordered reflexive, strictly convex, and smooth Banach space with respect to a minihedral cone $P$ and satisfy condition $\left(\mathrm{H}_{2}\right)$. Suppose that $C$ is closed convex subminihedral set of $X$. Moreover, $C$ satisfies the condition:

$$
\left(H_{3}\right)\|x \wedge y\|^{2}+\|x \vee y\|^{2} \leq\|x\|^{2}+\|y\|^{2}, \forall x, y \in C .
$$

Then, $\Pi_{C}$ is increasing.

Remark 6. The minihedral cones of many Banach spaces satisfy $\left(H_{3}\right)$. For example, if $p \geq 2$, every subminihedral set $M$ of $\left(\ell^{p}, \preceq\right)$ (here partial order $\preceq$ is defined coordinatewise) such that $x \geq \theta, \forall x \in M$, then $M$ satisfies $\left(H_{3}\right)$; if $p \geq 2$, every subminihedral set $M$ of $\left(R^{n, p}, \preceq\right.$ ) (here $\preceq$ stands again for the coordinatewise ordering), such that $x \geq \theta, \forall x \in M$, then $\mathrm{M}$ satisfies $\left(\mathrm{H}_{3}\right)$. See [5] for more details.

Lemma 7. If $X$ is a uniformly convex and smooth Banach space and $C$ is a nonempty, closed, and convex subset of $X$, then the generalized projection operator $\Pi_{C}: X \rightarrow C$ is continuous.

Proof. Since $X$ is a uniformly convex and smooth Banach space, $\Pi_{C}$ is single valued. Suppose $x_{n} \rightarrow x$, as $n \rightarrow \infty$, and suppose $\Pi_{C}\left(x_{n}\right)=\widehat{x}_{n}(n=1,2,3, \ldots)$, and $\Pi_{C}(x)=\hat{x}$. From the inequalities

$$
\begin{aligned}
\left(\left\|x_{n}\right\|-\left\|\widehat{x}_{n}\right\|\right)^{2} & \leq W\left(x_{n}, \widehat{x}_{n}\right) \\
& \leq W\left(x_{n}, \widehat{x}\right) \\
& \leq\left(\left\|x_{n}\right\|+\|\widehat{x}\|\right)^{2}
\end{aligned}
$$

and the hypothesis that $x_{n} \rightarrow x$, as $n \rightarrow \infty$, it yields $\left\{\widehat{x}_{n}\right\}$ is a bounded subset of $X$. Since $X$ is reflexive, there exists a subsequence of $\left\{\widehat{x}_{n}\right\}$; without loss of the generality, we may assume it is itself, such that $\left\{\widehat{x}_{n}\right\}$ converges weakly to $x^{\prime}$. From the properties of weakly convergence, we have $\left\|x^{\prime}\right\| \leq \liminf _{n \rightarrow \infty}\left\|\widehat{x}_{n}\right\|$. Moreover, $W(x, \widehat{x}) \leq W\left(x, \widehat{x}_{n}\right)$ and $W\left(x_{n}, \widehat{x}_{n}\right) \leq W\left(x_{n}, \widehat{x}\right)$, which implies $W\left(x, \widehat{x}_{n}\right) \rightarrow W(x, \widehat{x})$, as $n \rightarrow \infty$. Now we have

$$
\begin{aligned}
W\left(x, x^{\prime}\right) & =\|x\|^{2}-2\left\langle J x, x^{\prime}\right\rangle+\left\|x^{\prime}\right\|^{2} \\
& =\lim _{n \rightarrow \infty}\left(\|x\|^{2}-2\left\langle J x, \widehat{x}_{n}\right\rangle+\left\|x^{\prime}\right\|^{2}\right) \\
& \leq \liminf _{n \rightarrow \infty}\left(\|x\|^{2}-2\left\langle J x, \widehat{x}_{n}\right\rangle+\left\|\widehat{x}_{n}\right\|^{2}\right) \\
& =\liminf _{n \rightarrow \infty} W\left(x, \widehat{x}_{n}\right) \\
& =\lim _{n \rightarrow \infty} W\left(x, \widehat{x}_{n}\right) \\
& =\inf _{y \in C} W(x, y) .
\end{aligned}
$$

Thus we have $x^{\prime}=\widehat{x}$.

For any $\lambda \in[0,1]$, one has $\lambda \hat{x}+(1-\lambda) \widehat{x}_{n} \in C$. From the inequality $W(x, \widehat{x}) \leq W\left(x, \lambda \widehat{x}+(1-\lambda) \widehat{x}_{n}\right)$, we have

$$
\begin{aligned}
& \|x\|^{2}-2\langle J x, \widehat{x}\rangle+\|\widehat{x}\|^{2} \leq\|x\|^{2} \\
& \quad \leq\|x\|^{2}-2\left\langle J x, \lambda \hat{x}+(1-\lambda) \widehat{x}_{n}\right\rangle+\left\|\lambda \hat{x}+(1-\lambda) \widehat{x}_{n}\right\|^{2} .
\end{aligned}
$$

Therefore,

$$
2\left\langle J x,(1-\lambda)\left(\widehat{x}_{n}-\widehat{x}\right)\right\rangle \leq\left\|\lambda \widehat{x}+(1-\lambda) \widehat{x}_{n}\right\|^{2}-\|\widehat{x}\|^{2} .
$$

Similar to the above argument, from inequality $W\left(x_{n}, \widehat{x}_{n}\right) \leq$ $W\left(x_{n}, \widehat{x}\right)$, we obtain

$$
2\left\langle J x_{n}, \widehat{x}-\widehat{x}_{n}\right\rangle \leq\|\widehat{x}\|^{2}-\left\|\widehat{x}_{n}\right\|^{2} .
$$

Adding the above two inequalities side by side, we obtain

$$
\begin{aligned}
2\langle J x & \left.-J x_{n}, \widehat{x}_{n}-\hat{x}\right\rangle \\
\leq & \left\|\lambda \widehat{x}+(1-\lambda) \widehat{x}_{n}\right\|^{2} \\
& -\left\|\widehat{x}_{n}\right\|^{2}+2 \lambda\left\langle J x, \widehat{x}_{n}-\widehat{x}\right\rangle \\
\leq & \lambda^{2}\|\widehat{x}\|^{2} \\
& +2 \lambda(1-\lambda)\|\widehat{x}\|\left\|\widehat{x}_{n}\right\|+(1-\lambda)^{2}\left\|\widehat{x}_{n}\right\|^{2}
\end{aligned}
$$




$$
\begin{aligned}
& -\left\|\widehat{x}_{n}\right\|^{2}+2 \lambda\left\langle J x, \widehat{x}_{n}-\widehat{x}\right\rangle \\
\leq & \lambda^{2}\|\widehat{x}\|^{2}+\lambda(1-\lambda)\left(\|\widehat{x}\|^{2}+\left\|\widehat{x}_{n}\right\|^{2}\right) \\
& +(1-\lambda)^{2}\left\|\widehat{x}_{n}\right\|^{2}-\left\|\widehat{x}_{n}\right\|^{2}+2 \lambda\left\langle J x, \widehat{x}_{n}-\widehat{x}\right\rangle \\
= & \lambda\left(\|\widehat{x}\|^{2}-\left\|\widehat{x}_{n}\right\|^{2}\right)+2 \lambda\left\langle J x, \widehat{x}_{n}-\widehat{x}\right\rangle .
\end{aligned}
$$

So

$$
2\left\langle J x-J x_{n}, \widehat{x}-\widehat{x}_{n}\right\rangle \geq \lambda\left(\left\|\widehat{x}_{n}\right\|^{2}-\|\widehat{x}\|^{2}\right)+2 \lambda\left\langle J x, \widehat{x}-\widehat{x}_{n}\right\rangle .
$$

If we use the inequalities $W(x, \widehat{x}) \leq W\left(x, \widehat{x}_{n}\right)$ and $W\left(x_{n}, \widehat{x}_{n}\right) \leq W\left(x_{n}, \lambda \widehat{x}+(1-\lambda) \widehat{x}_{n}\right)$, similar to the above argument, we obtain

$$
\begin{aligned}
2\left\langle J x-J x_{n}, \widehat{x}-\widehat{x}_{n}\right\rangle \geq & (1-\lambda)\left(\|\widehat{x}\|^{2}-\left\|\widehat{x}_{n}\right\|^{2}\right) \\
& +2(1-\lambda)\left\langle J x_{n}, \widehat{x}_{n}-\widehat{x}\right\rangle .
\end{aligned}
$$

In (18) and (19), taking $\lambda=1 / 2$, we have

$$
\begin{aligned}
& 4\left\langle J x-J x_{n}, \widehat{x}-\widehat{x}_{n}\right\rangle \geq\left(\left\|\widehat{x}_{n}\right\|^{2}-\|\widehat{x}\|^{2}\right)+2\left\langle J x, \widehat{x}-\widehat{x}_{n}\right\rangle, \\
& 4\left\langle J x-J x_{n}, \widehat{x}-\widehat{x}_{n}\right\rangle \geq\left(\|\widehat{x}\|^{2}-\left\|\widehat{x}_{n}\right\|^{2}\right)+2\left\langle J x_{n}, \widehat{x}_{n}-\widehat{x}\right\rangle .
\end{aligned}
$$

From the conditions that $x_{n} \rightarrow x$, as $n \rightarrow \infty$ and $X$ is a smooth Banach space, we have $J x_{n} \rightarrow J x$, as $n \rightarrow \infty$. Using $\widehat{x}_{n} \rightarrow \widehat{x}$, as $n \rightarrow \infty$ and combining (20), it yields $\left\|\widehat{x}_{n}\right\| \rightarrow$ $\|\widehat{x}\|$, as $n \rightarrow \infty$. Since $X$ is a uniformly convex Banach space, then $X$ has the Kadec-Klee property. Therefore, we obtain $\widehat{x}_{n} \rightarrow \widehat{x}$, as $n \rightarrow \infty$. Thus this lemma is proved.

Lemma 8. Let $(X, \preceq)$ be a real partially ordered reflexive, strictly convex, and smooth Banach space with respect to $P$ and satisfy condition $\left(\mathrm{H}_{2}\right)$. Suppose that $P$ is a minihedral cone and satisfies the condition:

$$
\left(H_{4}\right)\|x \wedge y\|^{2}+\|x \vee y\|^{2} \leq\|x\|^{2}+\|y\|^{2}, \forall x \in X, \quad y \in P .
$$

Then, $\Pi_{P}$ is increasing, and $\Pi_{P}(x+y) \preceq \Pi_{P}(x)+\Pi_{P}(y)$, $\forall x, y \in X$.

Proof. Since $\left(H_{4}\right)$ implies $\left(H_{3}\right)$ and $P$ is subminihedral, from Lemma $5, \Pi_{P}$ is increasing. Next, we prove $x \preceq \Pi_{P}(x), \forall x \in$ $X$. To derive a contradiction, assume that there exists $x_{0}$ which does not satisfy $x_{0} \preceq \Pi_{P}\left(x_{0}\right)$; that is, $x_{0} \wedge \Pi_{P}\left(x_{0}\right) \neq x_{0}$ and $x_{0} \vee \Pi_{P}\left(x_{0}\right) \neq \Pi_{P}\left(x_{0}\right)$. Then we have

$$
W\left(x_{0}, \Pi_{P}\left(x_{0}\right)\right)<W\left(x_{0}, x_{0} \vee \Pi_{P}\left(x_{0}\right)\right) ;
$$

that is,

$$
\begin{aligned}
& \left\|x_{0}\right\|^{2}-2\left\langle J x_{0}, \Pi_{P}\left(x_{0}\right)\right\rangle+\left\|\Pi_{P}\left(x_{0}\right)\right\|^{2} \\
& \quad<\left\|x_{0}\right\|^{2}-2\left\langle J x_{0}, x_{0} \vee \Pi_{P}\left(x_{0}\right)\right\rangle+\left\|x_{0} \vee \Pi_{P}\left(x_{0}\right)\right\|^{2} .
\end{aligned}
$$

Hence,

$$
\begin{aligned}
& 2\left\langle J x_{0}, x_{0} \vee \Pi_{P}\left(x_{0}\right)-\Pi_{P}\left(x_{0}\right)\right\rangle \\
& \quad<\left\|x_{0} \vee \Pi_{P}\left(x_{0}\right)\right\|^{2}-\left\|\Pi_{P}\left(x_{0}\right)\right\|^{2} .
\end{aligned}
$$

As $x_{0} \wedge \Pi_{P}\left(x_{0}\right) \neq x_{0}$, we have

$$
\begin{gathered}
W\left(x_{0}, x_{0} \wedge \Pi_{P}\left(x_{0}\right)\right)= \\
\quad\left\|x_{0}\right\|^{2}-2\left\langle J x_{0}, x_{0} \wedge \Pi_{P}\left(x_{0}\right)\right\rangle \\
+\left\|x_{0} \wedge \Pi_{P}\left(x_{0}\right)\right\|^{2}>0,
\end{gathered}
$$

and then,

$$
2\left\langle J x_{0}, x_{0} \wedge \Pi_{P}\left(x_{0}\right)\right\rangle<\left\|x_{0}\right\|^{2}+\left\|x_{0} \wedge \Pi_{P}\left(x_{0}\right)\right\|^{2} .
$$

Since $x_{0} \wedge \Pi_{P}\left(x_{0}\right)+x_{0} \vee \Pi_{P}\left(x_{0}\right)=x_{0}+\Pi_{P}\left(x_{0}\right)$, from (23) and (25), we have

$$
\begin{aligned}
2\left\langle J x_{0}, x_{0}\right\rangle< & \left\|x_{0}\right\|^{2}+\left\|x_{0} \wedge \Pi_{P}\left(x_{0}\right)\right\|^{2} \\
& +\left\|x_{0} \vee \Pi_{P}\left(x_{0}\right)\right\|^{2}-\left\|\Pi_{P}\left(x_{0}\right)\right\|^{2} .
\end{aligned}
$$

And hence $\left\|x_{0} \wedge \Pi_{P}\left(x_{0}\right)\right\|^{2}+\left\|x_{0} \vee \Pi_{P}\left(x_{0}\right)\right\|^{2}-\left\|\Pi_{P}\left(x_{0}\right)\right\|^{2}-$ $\left\|x_{0}\right\|^{2}>0$. This contradicts $\left(H_{4}\right)$. Thus, $x \preceq \Pi_{P}(x), \forall x \in X$. And hence,

$$
x+y \preceq \Pi_{P}(x)+\Pi_{P}(y), \quad \forall x, y \in X .
$$

As $\Pi_{P}$ is increasing, we have

$$
\Pi_{P}(x+y) \preceq \Pi_{P}(x)+\Pi_{P}(y), \quad \forall x, y \in X .
$$

The assertion is proved.

Lemma 9. Let $(X, \preceq)$ be a real partially ordered reflexive, strictly convex, and smooth Banach space with respect to a minihedral cone $P$ and satisfy condition $\left(\mathrm{H}_{2}\right)$. Suppose $u_{0}, v_{0} \in$ $X$ with $u_{0} \prec v_{0}$ and the following condition is satisfied:

$$
\begin{aligned}
& \left(H_{5}\right)\|x \wedge y\|^{2}+\|x \vee y\|^{2} \leq\|x\|^{2}+\|y\|^{2}, \forall x \in X, y \in \\
& {\left[u_{0}, v_{0}\right] .}
\end{aligned}
$$

Then, $\Pi_{\left[u_{0}, v_{0}\right]}$ is increasing, and

$$
\begin{array}{r}
\Pi_{\left[u_{0}, v_{0}\right]}(t x+(1-t) y) \preceq t \prod_{\left[u_{0}, v_{0}\right]}(x)+(1-t) \Pi_{\left[u_{0}, v_{0}\right]}(y) \\
\forall t \in[0,1], \quad \forall x, y \preceq v_{0} .
\end{array}
$$

Proof. Following a similar argument as in the proof of Lemma 8, we obtain that $\Pi_{\left[u_{0}, v_{0}\right]}$ is increasing and $x \preceq$ $\Pi_{\left[u_{0}, v_{0}\right]}(x), \forall x \preceq v_{0}$. And hence,

$$
\begin{array}{r}
t x+(1-t) y \preceq t \prod_{\left[u_{0}, v_{0}\right]}(x)+(1-t) \Pi_{\left[u_{0}, v_{0}\right]}(y), \\
\forall t \in[0,1], \quad x, y \preceq v_{0} .
\end{array}
$$

As $\Pi_{\left[u_{0}, v_{0}\right]}$ is increasing and $t \Pi_{\left[u_{0}, v_{0}\right]}(x)+(1-t) \Pi_{\left[u_{0}, v_{0}\right]}(y) \in$ $\left[u_{0}, v_{0}\right]$, we have

$$
\Pi_{\left[u_{0}, v_{0}\right]}(t x+(1-t) y) \preceq t \Pi_{\left[u_{0}, v_{0}\right]}(x)+(1-t) \Pi_{\left[u_{0}, v_{0}\right]}(y) .
$$

The proof is completed. 
Remark 10. If $\left(H,\|\cdot\|_{1}\right)$ is a partially ordered Hilbert space with respect to $P$ and $P$ a minihedral cone, $\left(H_{4}\right)$ and $\left(H_{5}\right)$ are satisfied.

From the above properties of the generalized projection operators and order-theoretic fixed point theorems, we can obtain the following best approximation theorems.

Theorem 11. Let $(X, \preceq)$ be a real partially ordered uniformly convex and smooth Banach space with respect to a minihedral cone $P$ and satisfy condition $\left(H_{2}\right)$. Suppose that $f:\left[u_{0}, v_{0}\right] \rightarrow$ $X$ is an increasing map. Moreover, $\left[u_{0}, v_{0}\right]$ satisfies the condition $\left(\mathrm{H}_{3}\right)$ and $f\left(\left[u_{0}, v_{0}\right]\right)$ is relatively compact. Then, $f$ has a minimum best approximation point $x_{*}$ and a maximum best approximation point $x^{*}$ with respect to $W(x, y)$ in $\left[u_{0}, v_{0}\right]$, such that

$$
\begin{gathered}
u_{0} \preceq u_{1} \preceq \cdots \preceq u_{n} \preceq \cdots \preceq x_{*} \preceq x^{*} \\
\preceq \cdots \preceq v_{n} \preceq \cdots \preceq v_{1} \preceq v_{0},
\end{gathered}
$$

where $u_{n}=\Pi_{\left[u_{0}, v_{0}\right]}\left(f\left(u_{n-1}\right)\right), v_{n}=\Pi_{\left[u_{0}, v_{0}\right]}\left(f\left(v_{n-1}\right)\right)(n=1,2$, $3, \ldots)$.

Proof. Define $F:\left[u_{0}, v_{0}\right] \rightarrow\left[u_{0}, v_{0}\right]$ by $F(x)=$ $\Pi_{\left[u_{0}, v_{0}\right]}(f(x))$. From Lemma 5, we get $F$ is increasing. It is easy to see $u_{0} \preceq F\left(u_{0}\right)$ and $F\left(v_{0}\right) \preceq v_{0}$. By Lemma 7 , we know $\Pi_{\left[u_{0}, v_{0}\right]}$ is continuous and $F\left(\left[u_{0}, v_{0}\right]\right)$ is relatively compact. Thus $F$ satisfies all conditions of Theorem 2.1.4 in [22]. Then, $F$ has a minimum fixed point $x_{*}$ and a maximum fixed point $x^{*}$ and satisfies (32). Now we consider $F\left(x_{*}\right)=x_{*}, F\left(x^{*}\right)=$ $x^{*}$; that is, $\Pi_{\left[u_{0}, v_{0}\right]}\left(f\left(x_{*}\right)\right)=x_{*}$ and $\Pi_{\left[u_{0}, v_{0}\right]}\left(f\left(x^{*}\right)\right)=x^{*}$. By the definition of $\Pi_{\left[u_{0}, v_{0}\right]}$, we get

$$
\begin{aligned}
W\left(f\left(x_{*}\right), x_{*}\right) & =\inf _{y \in\left[u_{0}, v_{0}\right]} W\left(f\left(x_{*}\right), y\right) \\
& =d_{W}\left(f\left(x_{*}\right),\left[u_{0}, v_{0}\right]\right), \\
W\left(f\left(x^{*}\right), x^{*}\right) & =\inf _{y \in\left[u_{0}, v_{0}\right]} W\left(f\left(x^{*}\right), y\right) \\
& =d_{W}\left(f\left(x^{*}\right),\left[u_{0}, v_{0}\right]\right) .
\end{aligned}
$$

The assertion is proved.

Theorem 12. Let $(X, \preceq)$ be a real partially ordered reflexive, strictly convex, and smooth Banach space with respect to a normal and minihedral cone $P$ and satisfy condition $\left(\mathrm{H}_{2}\right)$. Suppose that $f:\left[u_{0}, v_{0}\right] \rightarrow X$ is an increasing map. Moreover, $\left[u_{0}, v_{0}\right]$ satisfies the condition $\left(H_{3}\right)$. Then, $f$ has a minimum best approximation point $x_{*}$ and a maximum best approximation point $x^{*}$ with respect to $W(x, y)$ in $\left[u_{0}, v_{0}\right]$. Moreover, if $u_{n}=$ $\Pi_{\left[u_{0}, v_{0}\right]}\left(f\left(u_{n-1}\right)\right), v_{n}=\Pi_{\left[u_{0}, v_{0}\right]}\left(f\left(v_{n-1}\right)\right)(n=1,2,3, \ldots),(32)$ holds.

Proof. Define $F:\left[u_{0}, v_{0}\right] \rightarrow\left[u_{0}, v_{0}\right]$ by $F(x)=$ $\Pi_{\left[u_{0}, v_{0}\right]}(f(x))$. From Lemma 5 , we get $F$ is increasing. It is easy to see $u_{0} \preceq F\left(u_{0}\right)$ and $F\left(v_{0}\right) \preceq v_{0}$. Since $X$ is reflexive and $P$ is normal, $P$ is regular. Thus $F$ satisfies all conditions of Theorem 3.1.4 in [23]. Then, $F$ has a minimum fixed point $x_{*}$ and a maximum fixed point $x^{*}$ and satisfies (32). By the definition of $\Pi_{\left[u_{0}, v_{0}\right]}$, the assertion is proved.
Remark 13. In the above Theorem 11, $f$ is discontinuous map. And in Theorem 12, $f$ is discontinuous map and has no compact conditions.

Example 14. Let $(X, \preceq)=\left(\ell^{2}, \preceq\right)$. Here $\preceq$ stands for the coordinatewise ordering. It is easy to prove that all conditions in Theorem 12 hold. Given $u_{0}, v_{0} \in \ell^{2}$ such that $u_{0} \prec v_{0}$. Then, every increasing $f:\left[u_{0}, v_{0}\right] \rightarrow \ell^{2}$ has a minimum best approximation point and a maximum best approximation point with respect to $W(x, y)$ in $\left[u_{0}, v_{0}\right]$.

Theorem 15. Let $(X, \preceq)$ be a real partially ordered reflexive, strictly convex, and smooth Banach space with respect to $P$. If $u_{0} \prec v_{0}$ and the following conditions are satisfied,

(i) $P$ is a normal, minihedral cone with satisfying $\left(\mathrm{H}_{2}\right)$ and $\left(\mathrm{H}_{5}\right)$;

(ii) $f:\left[u_{0}, v_{0}\right] \rightarrow X$ is an increasing and convex map;

(iii) there exists $a 0<<1$ such that $f\left(v_{0}\right) \preceq \varepsilon u_{0}+(1-\varepsilon) v_{0}$, then, $f$ has a unique approximation point $\widehat{x}$ with respect to $W(x, y)$ in $\left[u_{0}, v_{0}\right]$. Moreover, if we take $x_{n}=\Pi_{\left[u_{0}, v_{0}\right]}\left(f\left(x_{n-1}\right)\right)(n=1,2,3, \ldots)$ for $\forall x_{0} \in\left[u_{0}, v_{0}\right]$,

$$
\begin{gathered}
\left\|x_{n}-\hat{x}\right\| \longrightarrow 0 \quad(n \longrightarrow \infty), \\
\left\|x_{n}-\hat{x}\right\| \leq M(1-\varepsilon)^{n} \quad(n=1,2,3, \ldots),
\end{gathered}
$$

where $M>0$ has nothing to do with $x_{0}$.

Proof. Define $F:\left[u_{0}, v_{0}\right] \rightarrow\left[u_{0}, v_{0}\right]$ by $F(x)=$ $\Pi_{\left[u_{0}, v_{0}\right]}(f(x))$. Since $f$ is convex and $\Pi_{\left[u_{0}, v_{0}\right]}$ is increasing, for $\forall t \in[0,1]$, we have

$$
\begin{aligned}
F(t x+(1-t) y) & =\Pi_{\left[u_{0}, v_{0}\right]}(f(t x+(1-t) y)) \\
& \preceq \Pi_{\left[u_{0}, v_{0}\right]}(t f(x)+(1-t) f(y)) .
\end{aligned}
$$

Using Lemma 9 and $f(x) \preceq f\left(v_{0}\right) \preceq v_{0}$, we obtain

$$
\begin{aligned}
F(t x+(1-t) y) & \leq t \Pi_{\left[u_{0}, v_{0}\right]}(f(x))+(1-t) \Pi_{\left[u_{0}, v_{0}\right]}(f(y)) \\
& =t F(x)+(1-t) F(y) .
\end{aligned}
$$

Thus $F$ is convex. And $F\left(v_{0}\right) \preceq \varepsilon u_{0}+(1-\varepsilon) v_{0}$. Thus $F$ satisfies all conditions of Theorem 3.1.6 in [23]. Then, $F$ has a unique fixed point $\widehat{x}$ and satisfies (35). By the definition of $\Pi_{\left[u_{0}, v_{0}\right]}$, the assertion is proved.

\section{Fixed Point Theorems}

In this section, we will prove some new fixed point theorems for non-self-maps by using results of Section 3.

Theorem 16. Let $(X, \preceq)$ be a real partially ordered uniformly convex and smooth Banach space with respect to a minihedral cone $P$ and satisfy condition $\left(H_{2}\right)$. Suppose that $f:\left[u_{0}, v_{0}\right] \rightarrow$ $X$ is an increasing map and $f\left(\left[u_{0}, v_{0}\right]\right)$ is relative compact. Moreover, $\left[u_{0}, v_{0}\right]$ satisfies the condition $\left(\mathrm{H}_{3}\right)$ and

$$
\left|\operatorname{co}\{x, f(x)\} \cap\left[u_{0}, v_{0}\right]\right| \geq 2, \quad \forall x \in\left[u_{0}, v_{0}\right] .
$$

Then, $f$ has at least one fixed point in $\left[u_{0}, v_{0}\right]$. 
Proof. By Theorem 11, $f$ has at least one best approximation point $\widehat{x}$ in $\left[u_{0}, v_{0}\right]$; that is, $\Pi_{\left[u_{0}, v_{0}\right]}(f(\widehat{x}))=\widehat{x}$. From (11), we have

$$
\langle J(f(\widehat{x}))-J \widehat{x}, \widehat{x}-y\rangle \geq 0, \quad \forall y \in\left[u_{0}, v_{0}\right] .
$$

We may use (38) to find a $\lambda \in(0,1]$ such that $(1-\lambda) \widehat{x}+\lambda f(\widehat{x}) \in$ $\left[u_{0}, v_{0}\right]$, and hence

$$
\langle J(f(\widehat{x}))-J \widehat{x}, \widehat{x}-[(1-\lambda) \hat{x}+\lambda f(\widehat{x})]\rangle \geq 0 ;
$$

that is,

$$
\langle J(f(\widehat{x}))-J \widehat{x}, \widehat{x}-f(\widehat{x})\rangle \geq 0 .
$$

Moreover,

$$
\begin{aligned}
\langle J & (f(\hat{x}))-J \widehat{x}, f(\widehat{x})-\widehat{x}\rangle \\
& =\|f(\widehat{x})\|^{2}-\langle J(f(\widehat{x})), \widehat{x}\rangle-\langle J \widehat{x}, f(\widehat{x})\rangle+\|\widehat{x}\|^{2} \\
& \geq\|f(\widehat{x})\|^{2}-2\|f(\widehat{x})\|\|\widehat{x}\|+\|\widehat{x}\|^{2} \\
& =(\|f(\widehat{x})\|-\|\widehat{x}\|)^{2} \geq 0 .
\end{aligned}
$$

So we conclude that $\langle J(f(\widehat{x}))-J \widehat{x}, f(\widehat{x})-\widehat{x}\rangle=0$. It follows that $\|f(\hat{x})\|=\|\hat{x}\|$. Moreover, as $\langle J(f(\hat{x})), \hat{x}\rangle \leq\|f(\hat{x})\|\|\hat{x}\|$, and the inequality above must hold as an equality. We have $\langle J(f(\hat{x})), \widehat{x}\rangle=\|f(\widehat{x})\|\|\widehat{x}\|$. Therefore, $J(f(\widehat{x}))=J \widehat{x}$. And thus $f(\widehat{x})=\widehat{x}$. The assertion is proved.

Following a similar argument as in the proof of Theorem 16, we can obtain the following fixed point theorems.

Theorem 17. Let $(X, \preceq)$ be a real partially ordered uniformly convex and smooth Banach space with respect to $P$ and satisfy condition $\left(\mathrm{H}_{2}\right)$. Suppose that $P$ is a normal, minihedral cone and $f:\left[u_{0}, v_{0}\right] \rightarrow X$ is an increasing map. Moreover, $\left[u_{0}, v_{0}\right]$ satisfies the condition $\left(H_{3}\right)$ and (38). Then, $f$ has at least one fixed point in $\left[u_{0}, v_{0}\right]$.

Example 18. Let $(X, \preceq)=\left(L^{2}(\Omega), \preceq\right)$, the space of measurable functions which are the 2 nd power summable on $\Omega$. Endow $L^{2}(\Omega)$ with the following norm and the cone $P$ :

$$
\begin{gathered}
\|x\|=\left(\int_{\Omega}|x(t)|^{2} d \mu\right)^{1 / 2}, \\
P=\left\{x \in L^{2}(\Omega): x(t) \geq 0, \forall \text { a.e. } t \in \Omega\right\} .
\end{gathered}
$$

Given $u_{0}, v_{0} \in L^{2}(\Omega)$ such that $u_{0} \prec v_{0}$. It is easy to see that $\left(L^{2}(\Omega), \preceq\right)$ satisfies $\left(H_{2}\right)$ and $\left(H_{3}\right)$ holds in $\left[u_{0}, v_{0}\right]$. Thus, by Theorem 17 , every increasing $f:\left[u_{0}, v_{0}\right] \rightarrow L^{2}(\Omega)$ satisfying (38) has at least one fixed point in $\left[u_{0}, v_{0}\right]$.

Theorem 19. Let $(X, \preceq)$ be a real partially ordered reflexive, strictly convex, and smooth Banach space with respect to $P$. If $u_{0} \prec v_{0}$ and the following conditions are satisfied,

(i) $P$ is a normal, minihedral cone with satisfying $\left(\mathrm{H}_{2}\right)$, $\left(\mathrm{H}_{5}\right)$ and (38); (ii) $f:\left[u_{0}, v_{0}\right] \rightarrow X$ is an increasing and convex map;

(iii) there exists $0<\varepsilon<1$ such that $f\left(v_{0}\right) \preceq \varepsilon u_{0}+(1-\varepsilon) v_{0}$,

then, $f$ has a unique fixed point $\hat{x}$ in $\left[u_{0}, v_{0}\right]$. Moreover, if we take $x_{n}=\Pi_{\left[u_{0}, v_{0}\right]}\left(f\left(x_{n-1}\right)\right)(n=1,2,3, \ldots)$ for $\forall x_{0} \in\left[u_{0}, v_{0}\right]$,

$$
\begin{gathered}
\left\|x_{n}-\hat{x}\right\| \longrightarrow 0 \quad(n \longrightarrow \infty), \\
\left\|x_{n}-\widehat{x}\right\| \leq M(1-\varepsilon)^{n} \quad(n=1,2,3, \ldots),
\end{gathered}
$$

where $M>0$ has nothing to do with $x_{0}$.

\section{Conflict of Interests}

The authors declare that there is no conflict of interests regarding the publication of this paper.

\section{Acknowledgments}

Dezhou Kong and Lishan Liu were supported financially by the National Natural Science Foundation of China (11371221), the Specialized Research Foundation for the Doctoral Program of Higher Education of China (20123705110001), and the Program for Scientific Research Innovation Team in Colleges and Universities of Shandong Province. Yonghong Wu was supported financially by the Australian Research Council through an ARC Discovery Project Grant.

\section{References}

[1] Y. Alber, "Generalized projection operators in Banach spaces: properties and applications," in Proceedings of the Israel Seminar Ariel, Israel, Function Differential Equation, vol. 1, pp. 1-21, 1994.

[2] J. Li, "The generalized projection operator on reflexive Banach spaces and its applications," Journal of Mathematical Analysis and Applications, vol. 306, no. 1, pp. 55-71, 2005.

[3] G. Isac, "On the order monotonicity of the metric projection operator," in Approximation Theory, Wavelets and Applications, S. P. Singh, Ed., vol. 454 of NATO Science Series, pp. 365379, Kluwer Academic Publishers, Dordrecht, The Netherlands, 1995.

[4] H. Nishimura and E. A. Ok, "Solvability of variational inequalities on Hilbert lattices," Mathematics of Operations Research, vol. 37, no. 4, pp. 608-625, 2012.

[5] J. Li and E. A. Ok, "Optimal solutions to variational inequalities on Banach lattices," Journal of Mathematical Analysis and Applications, vol. 388, no. 2, pp. 1157-1165, 2012.

[6] K. Fan, "Extensions of two fixed point theorems of F. E. Browder," Mathematische Zeitschrift, vol. 112, pp. 234-240, 1969.

[7] T. Lin and S. Park, "Approximation and fixed-point theorems for condensing composites of multifunctions," Journal of Mathematical Analysis and Applications, vol. 223, no. 1, pp. 1-8, 1998.

[8] D. O'Regan and N. Shahzad, "Approximation and fixed point theorems for countable condensing composite maps," Bulletin of the Australian Mathematical Society, vol. 68, no. 1, pp. 161168, 2003.

[9] K. Tan and X. Yuan, "Random fixed-point theorems and approximation in cones," Journal of Mathematical Analysis and Applications, vol. 185, no. 2, pp. 378-390, 1994. 
[10] L. Liu, "Approximation theorems and fixed point theorems for various classes of 1-set-contractive mappings in Banach spaces," Acta Mathematica Sinica, vol. 17, no. 1, pp. 103-112, 2001.

[11] L. Liu, "Random approximations and random fixed point theorems for random 1-set-contractive non-self-maps in abstract cones," Stochastic Analysis and Applications, vol. 18, no. 1, pp. 125-144, 2000.

[12] L. Liu, "Some random approximations and random fixed point theorems for 1-set-contractive random operators," Proceedings of the American Mathematical Society, vol. 125, no. 2, pp. 515521, 1997.

[13] L. Liu, "Random approximations and random fixed point theorems in infinite-dimensional Banach spaces," Indian Journal of Pure and Applied Mathematics, vol. 28, no. 2, pp. 139-150, 1997.

[14] I. Beg and N. Shahzad, "Random fixed points of random multivalued operators on Polish spaces," Nonlinear Analysis: Theory, Methods \& Applications, vol. 20, no. 7, pp. 835-847, 1993.

[15] N. Shahzad, "Fixed point and approximation results for multimaps in S-KKM class," Nonlinear Analysis: Theory, Methods \& Applications, vol. 56, no. 6, pp. 905-918, 2004.

[16] J. Markin and N. Shahzad, "Best approximation theorems for nonexpansive and condensing mappings in hyperconvex spaces," Nonlinear Analysis. Theory, Methods \& Applications, vol. 70, no. 6, pp. 2435-2441, 2009.

[17] A. Amini-Harandi, "Best and coupled best approximation theorems in abstract convex metric spaces," Nonlinear Analysis: Theory, Methods \& Applications, vol. 74, no. 3, pp. 922-926, 2011.

[18] D. Roux and S. P. Singh, "On some fixed point theorems," International Journal of Mathematics and Mathematical Sciences, vol. 12, no. 1, pp. 61-64, 1989.

[19] L. Liu, "On approximation theorems and fixed point theorems for non-self-mappings in infinite-dimensional Banach spaces," Journal of Mathematical Analysis and Applications, vol. 188, no. 2, pp. 541-551, 1994.

[20] L. Liu and X. Li, "On approximation theorems and fixed point theorems for non-self-mappings in uniformly convex Banach spaces," Banyan Mathematical Journal, vol. 4, pp. 11-20, 1997.

[21] D. O'Regan, "Existence and approximation of fixed points for multivalued maps," Applied Mathematics Letters, vol. 12, no. 6, pp. 37-43, 1999.

[22] D. Guo and V. Lakshmikantham, Nonlinear Problems in Abstract Cones, Academic Press, New York, NY, USA, 1988.

[23] D. Guo, Y. Cho, and J. Zhu, Partial Ordering Methods in Nonlinear Problems, Nova Science Publishers, New York, NY, USA, 2004.

[24] P. Meyer-Nieberg, Banach Lattices, Universitext, Springer, New York, NY, USA, 1991.

[25] I. Cioranescu, "Geometry of Banach spaces," in Duality Mappings and Nonlinear Problems, Kluwer Academic Publishers, Dordrecht, The Netherlands, 1990.

[26] W. Takahashi, Nonlinear Functional Analysis, Fixed Point Theory and its Applications, Yokohama, Yokohama, Japan, 2000.

[27] D. Kong, L. Liu, and Y. Wu, "Best approximation and fixed-point theorems for discontinuous increasing maps in Banach lattices," Fixed Point Theory and Applications, vol. 2014, article 18, 2014. 


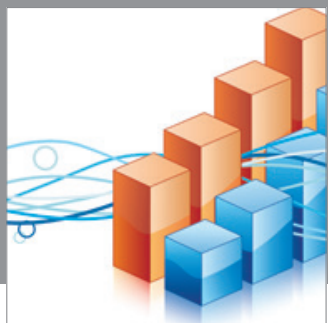

Advances in

Operations Research

mansans

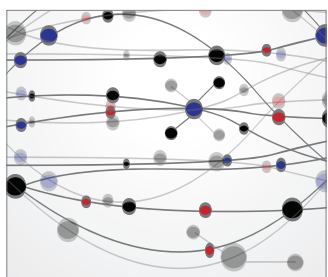

The Scientific World Journal
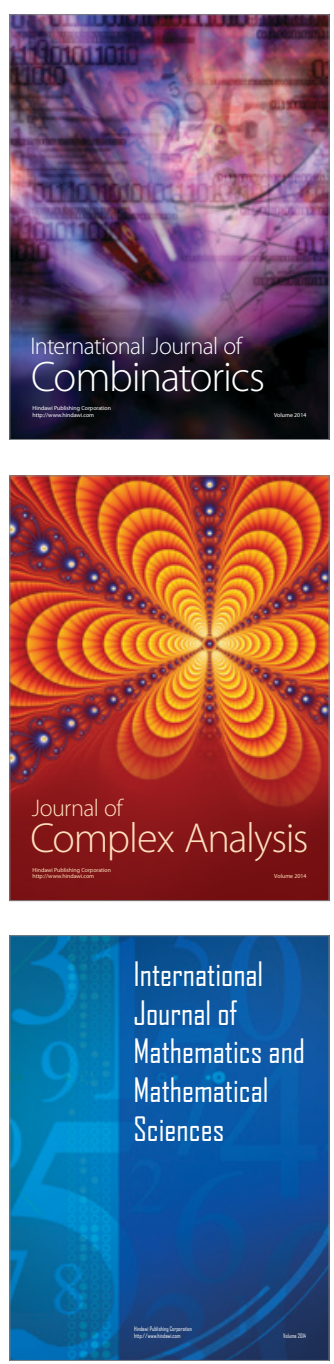
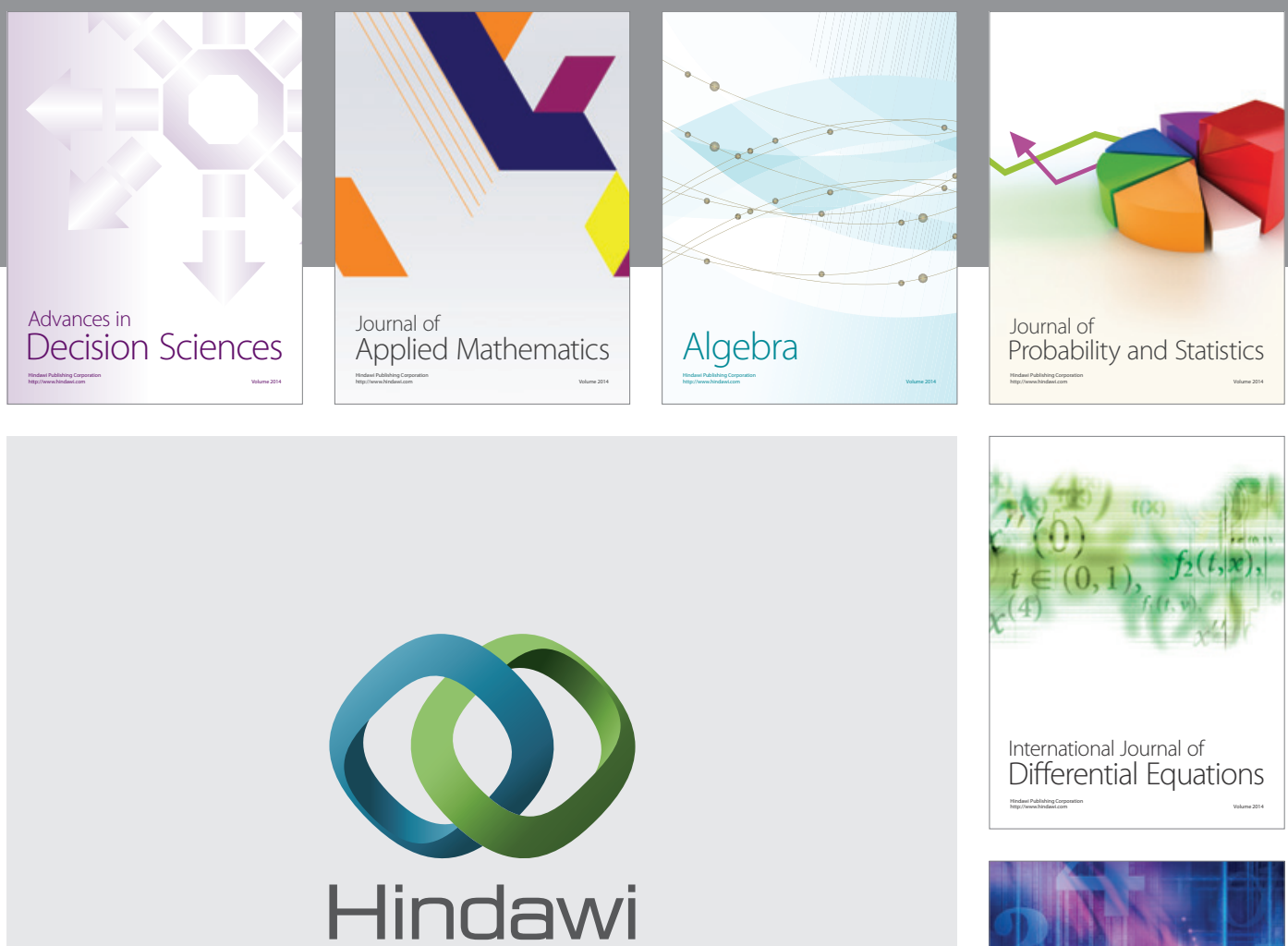

Submit your manuscripts at http://www.hindawi.com
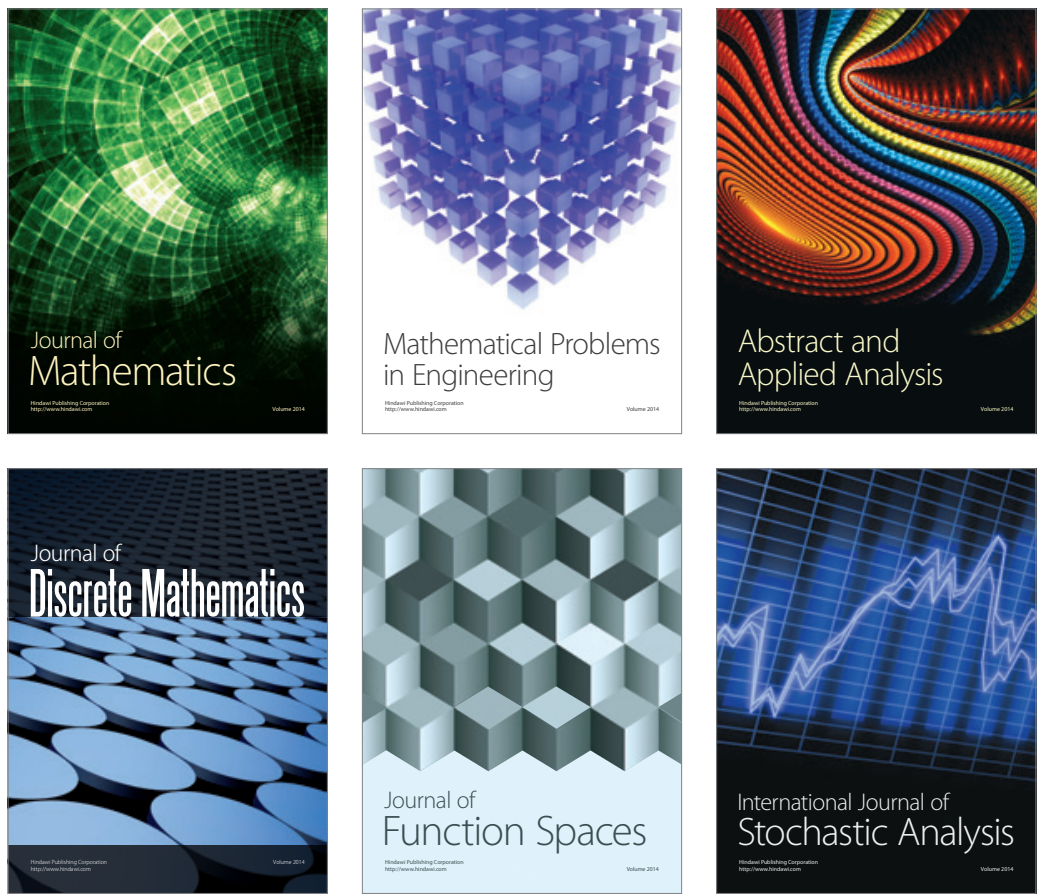

Journal of

Function Spaces

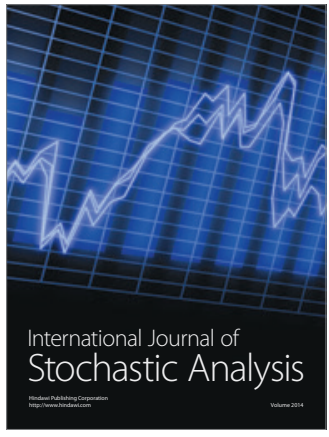

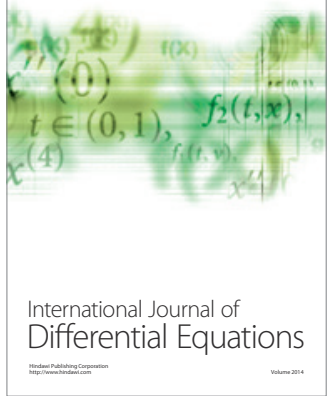
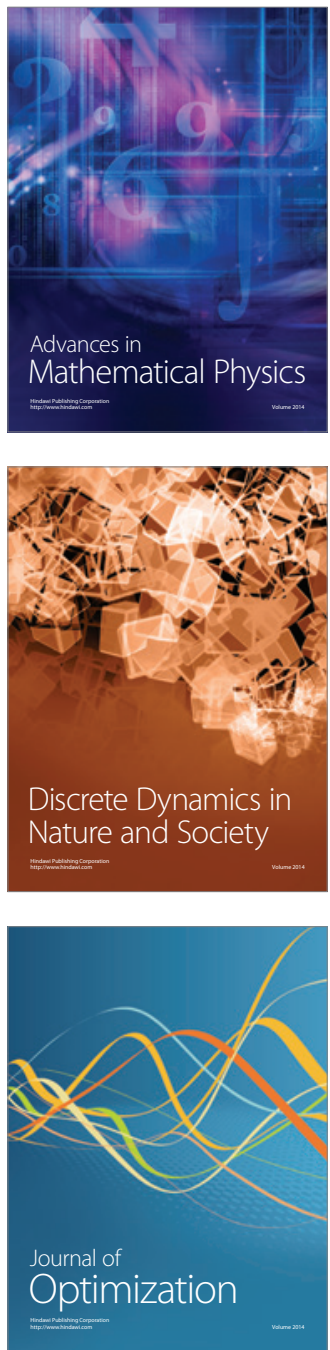